\title{
Depressionen: Diskurs zwischen Umwelt, Genen und Synapsen?
}

$\mathrm{D}$ ie Fortschritte in der Molekularbiologie und Computertechnologie haben in den letzten Jahren den Fokus der Erforschung seelischer Störungen von der differenzierten Beschreibung der Psychopathologie, von Epidemiologie, Psychodynamik und Verlauf hin zum Verständnis der somatischen Basis verlagert. Ein besonderer Schwerpunkt liegt dabei - neben der Neurobiologie - auch im Verständnis der Interaktion neuronaler Prozesse mit peripheren metabolischen Abläufen, die Auswirkungen auf das vaskuläre Risikoprofil der Betroffenen und damit die Lebenserwartung haben.

Affektive Störungen gehören zu den häufigsten psychiatrischen Erkrankungen in der Primärversorgung. Auf der Zellebene sprechen ältere neurochemische Befunde für eine Störung in der serotonergen bzw. noradrenergen Neurotransmission (Monoamin-Hypothese). Neuroendokrinologische Daten weisen außerdem auf eine Regulationsstörung der Hypothalamus-Hypophysen-Nebennierenrinden-Achse mit Hyperkortisolismus sowie auf Störungen des peripheren Lipidmetabolismus, der Insulinresistenz sowie der Schilddrüsenfunktion hin. Tierexperimentelle Befunde sprechen für Veränderungen der synaptischen sowie glialen Plastizität sowie der Neuroneogenese. Auch ein Mangel an Neurotrophinen ist seit kurzem beschrieben.

Diese Befunde haben die vorbestehenden Modelle zur Pathogenese der Depressionen dahingehend erweitert, dass depressive Störungen nicht primär nur ein Monoamin-Defizit darstellen, sondern Ausdruck gestörter neuronaler Plastizität mit fortlaufend verändertem „Dialog zwischen Synapsen und Genen“ sind. Über den Mangel an Monoaminen, dem Fehlen neurotropher Faktoren wie z.B. BDNF, Neuropeptidveränderungen, der Inhibition der Neuroneogenese sowie der gestörten Bildung von Transkriptionsfaktoren auch durch z.B. Kortisol („chronischer Stress“) verliert das Gehirn vorübergehend seine plastische Fähigkeit zur notwendigen Anpassung an die Herausforderungen der Umwelt. Dies führt in der Summation mit Vulnerabilitätsgenen (z.B. SerotoninTransporter-Gen), frühkindlicher Traumatisierung, Umweltfaktoren, gestörter Regulation der Stresssysteme und unzureichenden Bewältigungsstrategien auf der Verhaltensebene zu dem bekannten klinischen Phänomen einer depressiven Störung.

Auf der Systemebene sind Gemütserkrankungen korreliert mit Störungen der Interaktion von spezifischen Hirnarealen, deren Entdeckung auf histomorphologischen Befunden sowie insbesondere auf den Ergebnissen funktionell-bildgebender Verfahren beruht. Darauf aufbauend wurden in den letzten Jahren Netzwerkmodelle zur Affektmodulation und zur depressiven Störung entwickelt. Danach kommt den rostralen Anteilen des anterioren Gyrus Cinguli eine wichtige regulatorische Rolle bei der neuronalen Interaktion zwischen den dorsalen (Aufmerksamkeit, Kognition, Bewertung) und ventralen (Vitalfunktionen, Vegetativum, Affekte) Hirnanteilen zu. Eine depressive Störung ist assoziiert mit einer Störung der koordinierten Interaktion von entwicklungsgeschichtlich jüngeren, dorsalen und entwicklungsgeschichtlich älteren, ventralen kortikal-subkortikalen Regionen.

Im Rahmen dieses Schwerpunktsheftes soll den Lesern in diesem Kontext ein Überblick über neuere Aspekte zur Pathophysiologie und medikamentösen Therapie der Depressionen gegeben werden. Kritisch wird von Eberhard Fuchs und Gabriele Flügge (Göttingen) auf der Zellebene die Depression als Störung der Neuroplastizität diskutiert, die bildgebenden Befunde auf der Systemebene werden von Christian Vollmert und Dieter F. Braus (Hamburg) referiert, und Daniel Kopf und Michael Deuschle (Mannheim) sensibilisieren für die Bedeutung der peripheren Störung des Lipidmetabolismus und der Insulinresistenz. Abschließend werden darauf aufbauend praktische Hinweise zur Optimierung der aktuellen medikamentösen Therapiestrategien von Stephanie Krüger (Dresden) gegeben.

Zukünftige Untersuchungen werden zeigen, inwieweit sich diese Entwicklungen eignen, biologische Subgruppen unter den affektiven Störungen besser zu identifizieren, das Risikoprofil der Betroffenen zu reduzieren und die therapeutischen Optionen im Einzelfall in Ergänzung zur Psychotherapie weiter zu verbessern.

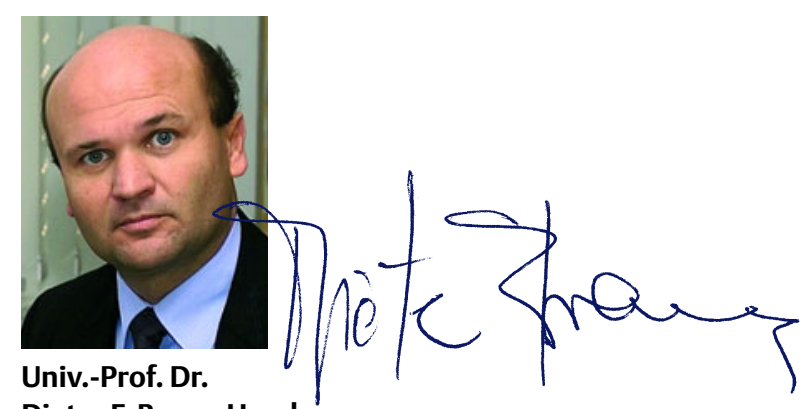

